\title{
Energy-Aware Routing for Wireless Sensor Networks by AHP
}

\author{
Xiaoling Wu, Jinsung Cho, Brian J. d'Auriol, and Sungyoung Lee* \\ Department of Computer Engineering, Kyung Hee University, Korea \\ \{xiaoling, dauriol, sylee\}@oslab.khu.ac.kr, chojs@khu.ac.kr
}

\begin{abstract}
Wireless sensor networks (WSNs) are comprised of energy constrained nodes. This limitation has led to the crucial need for energy-aware protocols to produce an efficient network. In this paper, we propose an energy aware geographical multipath routing scheme for WSNs. The distance to the destination location, remaining battery capacity, and queue size of candidate sensor nodes in the local communication range are taken into consideration for next hop relay node selection, and Analytical Hierarchy Process (AHP) is applied for decision making. Simulation results show that this scheme can extend the network lifetime longer than the original geographical routing scheme which only considers distance to the destination location. Moreover, the proposed scheme can reduce the packet loss rate and link failure rate since the buffer capacity is considered.
\end{abstract}

Keywords: Sensor networks, AHP, routing, lifetime, energy.

\section{Introduction}

Wireless sensor networks (WSNs) are expected to be widely employed in various applications such as medical care, military, environmental monitoring and industry since they have high flexibility, low production costs, and scalability [1]. The sensor nodes can sense the physical environment in various modalities, including acoustic, temperature, seismic, and infrared, etc. In WSNs, there exist some challenges, for example,

- The routing path (link) failure may happen during data transmission because of collision, node dying out (no battery), node busy, or other accidents. Some applications require real time information and data, which means retransmission is not possible. This motivates us to design a multipath routing scheme for wireless sensor networks.

- There exists energy constraint in WSNs because most sensors are battery operated. This motivates us to consider energy aware routing.

Many routing protocols have been developed for ad hoc networks, which can be summarized into two categories: table-driven (e.g., destination sequenced distance

\footnotetext{
${ }^{*}$ Corresponding author.
} 
vector [2], cluster switch gateway routing [3]) and source-initiated on-demand (e.g., ad hoc on-demand distance vector routing [4], dynamic source routing (DSR) [5]). In [6], Lee and Gerla propose a Split Multipath Routing protocol that builds maximal disjoint paths, where data traffic is distributed in two roots per session to avoid congestion and to use network resources efficiently. A Multipath Source Routing (MSR) scheme is proposed in [7], which is an extension of DSR. Their work focuses on distributing load adaptively among several paths. Nasipuri and Das [8] present the OnDemand Multipath Routing scheme which is also an extension of DSR. In their scheme, alternative routes are maintained, which can be utilized when the primary one fails.

In sensor networks, location is often more important than a specific node ID. For example, in sensor networks for target tracking, the target location is much more important than the ID of reporting node. Therefore, some location-aware routing schemes have been proposed for WSNs. A greedy geographic forwarding with limited flooding to circumvent the voids inside the network is proposed in [9], and some properties of greedy geographic routing algorithms are studied in [10]. Jain et al [11] proposes a geographical routing using partial information for WSNs.

In this paper, we propose an Analytical Hierarchy Process (AHP) based Energyaware Geographical Multipath Routing (AE-GMR) scheme for WSNs, and compare with Geographical Multipath Routing (GMR) scheme.

The rest of the paper is organized as follows. We define the basic assumptions and state the problems in section 2. The third section presents the proposed AHP based AE-GMR scheme. Section 4 evaluates and analyzes the performance of the proposed method. Finally, we draw the conclusion and discuss future work in section 5.

\section{Problem Statements}

In this paper, we also investigate the multipath routing problem and propose an AHP based Energy-aware Geographical Multipath Routing (AE-GMR) scheme. In the existing geographical routing approach (e.g., [11]), the path selection doesn't consider the remaining battery capacity of each node, which is a very important factor for energy efficiency and network lifetime. In our AE-GMR, we consider distance to the destination, remaining battery capacity, and queue size of each sensor node. Our scheme is a fully distributed approach where each sensor only needs the above three parameters, and we use AHP to handle these three parameters in the AE-GMR.

\section{A. Energy Model}

We adopt the same radio model as stated in [12] with $\varepsilon_{f_{s}}=10 \mathrm{pJ} / \mathrm{bit} / \mathrm{m}^{2}$ as amplifier constant, $E_{\text {elec }}=50 \mathrm{~nJ} / \mathrm{bit}$ as the energy being dissipated to run the transmitter or receiver circuitry. It is assumed that the transmission between the nodes follows a second-order power loss model. The energy cost of transmission for common sensor nodes at distance $d$ in transmitting an $l$-bit data is calculated as:

$$
E_{T}(l, d)=l E_{\text {elec }}+l \varepsilon_{f s} d^{2}
$$

and to receive the message, the radio expends: 


$$
E_{R}(l)=l E_{\text {elec }}
$$

and the energy for data aggregation is set as $E_{D A}=5 \mathrm{~nJ} / \mathrm{bit}$.

\section{B. Design Criteria}

In our AE-GMR design, we set up three criteria for node selection, and they are:

1) Distance to Destination: Distance of a node to the destination. The geographical location of destination is known to the source node (as in [11]), and the physical location of each sensor node can be estimated easily if the locations of three sensor nodes (within a communication range) are known in wireless sensor network. The node with shorter distance to the destination is preferred to be selected.

2) Residual energy: Remaining battery of the sensor node. The initial energy is predefined. In addition, the energy consumption for transmission and reception can be calculated using Eq. (1) and Eq. (2).

3) Queue size: It indicates the buffer capacity at the node. This parameter helps avoid packet drops due to congestion at the receiver.

The optimized node selection in multipath routing is a multiple factors optimization problem and can be achieved using the AHP approach which is introduced in the next section.

\section{Node Selection in Multipath Routing by AHP}

In our AE-GMR for $M$-path routing, the source node select $M$ nodes in its communication range for the first hop relay. Assume there are $N(N>M)$ nodes in its communication range, nodes that are farther to the destination node than the source node are not considered. Choosing $M$ nodes from remaining eligible nodes is based on AHP (as will be described in detail). Starting the second hop, each node in the $M$-path selects its next hop node also using AHP.

The Analytical Hierarchy Process (AHP) [13] is a multiple criteria decisionmaking method which decomposes a complex problem into a hierarchy of simple sub problems (or factors), synthesizes their importance to the problem, and finds the best solution. In this paper, AHP is used to determine the nodes which are eligible to be selected as next hop relay. It is carried out in three steps:

Step 1: Collect information and formulate the next hop routing nodes selection problem as a decision hierarchy of independent factors.

Step 2: Calculate the relative local weights of decision factors or alternatives of each level.

Step 3: Synthesize the above results to achieve the overall weight of each alternative node and choose the nodes with largest weight as the eligible next hop relay nodes. 


\section{A. Structuring Hierarchy}

The goal of the decision "Next hop relay node selection" is at the top level of the hierarchy as shown in Fig. 1. The next level consists of the decision factors which are called criteria for this goal. At the bottom level there exist the $N$ alternative sensor nodes to be evaluated.

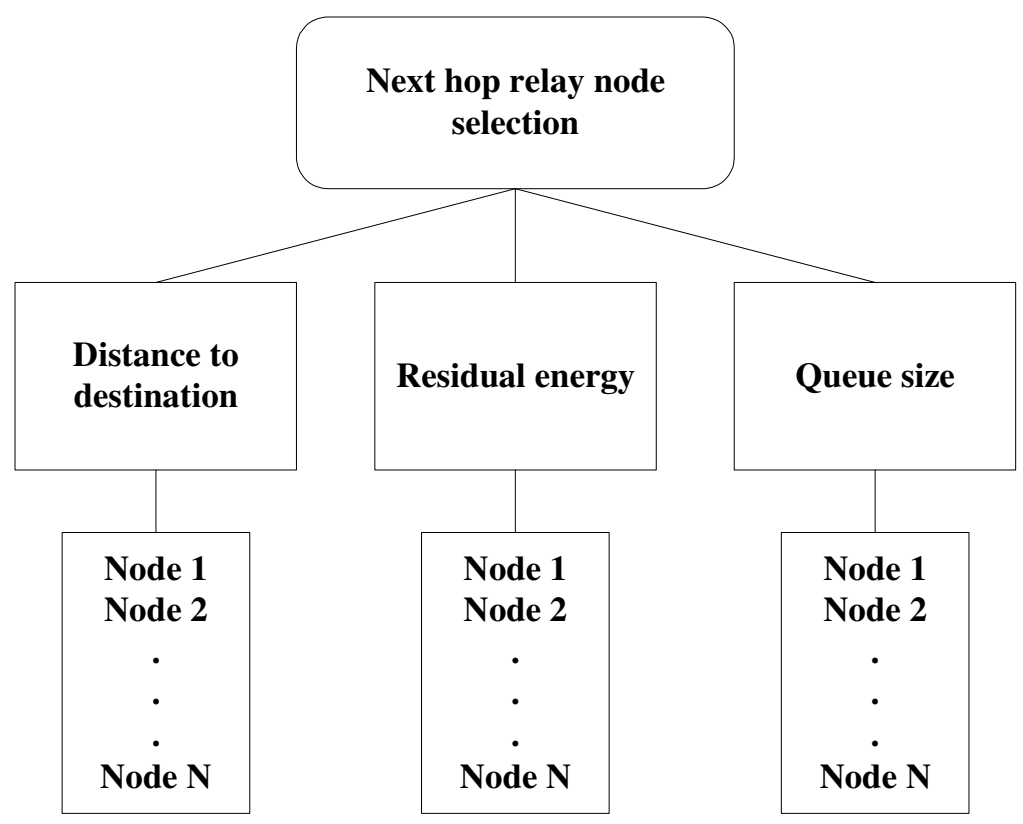

Fig. 1. AHP hierarchy for next hop relay nodes selection

\section{B. Calculating Local Weights}

Local weights consist of two parts: the weight of each decision factor to the goal and the weight of each nominee to each factor. Both of them are calculated with the same procedure. Taking the former as an example, we describe the procedure as the following three steps.

\section{1) Making Pairwise Comparison}

The evaluation matrices are built up through pairwise comparing each decision factor under the topmost goal. The comparison results are implemented by asking the questions: "Which is more important? How much?" and they may be presented in square matrix A as

$$
A=\left(a_{i j}\right)_{n \times n}=\left(\begin{array}{llll}
a_{11} & a_{12} & \hbar & a_{1 n} \\
a_{21} & a_{22} & \hbar & a_{2 n} \\
\hbar & \hbar & \hbar & \hbar \\
a_{n 1} & a_{n 2} & \hbar & a_{n n}
\end{array}\right),
$$


where $a_{i j}$ denotes the ratio of the $i^{\text {th }}$ factor weight to the $j^{\text {th }}$ factor weight, and $n$ is the number of factors. The fundamental 1 to 9 scale can be used to rank the judgments as shown in Table 1.

Table 1. A fundamental scale of 1 to 9

\begin{tabular}{cl}
\hline Number Rating & Verbal Judgment of Preferences \\
\hline 1 & Equally \\
3 & Moderately \\
5 & Strongly \\
7 & Very \\
9 & Extremely \\
\hline $2,4,6,8$ indicate the medium value of above pairwise comparison.
\end{tabular}

\section{2) Calculating Weight Vector}

For the given matrix A in Eq. (3), we calculate its eigenvalue equation written as AW $=\lambda_{\max } \mathrm{W}$, where $\mathrm{W}$ is non-zero vector called eigenvector, and $\lambda_{\max }$ is a scalar called eigenvalue. After standardizing the eigenvector $\mathrm{W}$, we regard the vector element of $\mathrm{W}$ as the local weight of each decision factor approximately, which can be denoted as:

$$
\mathbf{w}_{j}^{T}=\left\{w_{1}, w_{2}, \hbar, w_{n}\right\}
$$

\section{3) Checking for Consistency}

If every element in Eq. (3) satisfies the equations $a_{i j}=1 / a_{j i}$ and $a_{i k} \cdot a_{k j}=a_{i j}$, the matrix $\mathrm{A}$ is the consistency matrix. Unfortunately, the evaluation matrices are often not perfectly consistent due to people's random judgments. These judgment errors can be detected by a consistency ratio $(\mathrm{CR})$, which is defined as the ratio of consistency index $(\mathrm{CI})$ to random index (RI). CI can be achieved by

$$
C I=\left(\lambda_{\max }-n\right) /(n-1),
$$

where

$$
\lambda_{\max }=(1 / n) \sum_{i=1}^{n}(A W)_{i} / W_{i} .
$$

The $R I$ is given in Table 2 [14]. When $C R \leq 0.1$, the judgment errors are tolerable and the weight coefficients of the global weight matrix $W_{j}$ are the weights of decision factor under the topmost goal. Otherwise, the pairwise comparisons should be adjusted until matrix A satisfies the consistency check.

Table 2. Random index

\begin{tabular}{cccccccccccc}
\hline $\mathrm{n}$ & 1 & 2 & 3 & 4 & 5 & 6 & 7 & 8 & 9 & 10 & 11 \\
$\mathrm{RI}$ & 0 & 0 & 0.58 & 0.90 & 1.12 & 1.24 & 1.32 & 1.41 & 1.45 & 1.49 & 1.51 \\
\hline
\end{tabular}




\section{Calculating Global Weights}

From above steps, we can obtain not merely the weights of decision factors towards the topmost goal from $W_{j}$ but also the weights of alternative nodes towards each factor. If there are four candidate nodes in the communication range, all the four weight matrixes of alternatives under three factors construct a $4 \times 3$ matrix, denoted as $W_{n_{i} / j}$, $i=1,2, \ldots, 4, j=1,2,3$.

The global weight of each senor node can be achieved through multiplying the local weight by its corresponding parent. So the final weight matrix in the symbol of $W_{n_{i}}$ is calculated as

$$
W_{n_{i}}=W_{n_{i} / j} \cdot W_{j},
$$

where the final weight of each alternative is calculated as

$$
W_{n_{i}}=\sum_{j=1}^{3} W_{n_{i} / j} \cdot W_{j} .
$$

The larger the final weight of node, the higher the probability of node which is eligible to be selected. Thus, the $M$ nodes with the largest weight are selected as the next hop relay nodes in multipath routing.

In this paper, we assume that each sensor node keeps a table which has some information about its neighbor nodes: locations, battery level, and queue size. The table is updated periodically by the locally-broadcasted information (beacon) from each neighbor node. We define a time interval $T$, during which the three parameters (locations, battery level, and queue size) do not change very much. This time interval $T$ is the shortest time duration that a sensor node will send another beacon. Each sensor examines itself the status of the three parameters in every period $T$, and if a certain parameter has changed above a threshold, it will locally broadcast a beacon.

\section{Path Set Up}

In the route discovery phase, the source node uses AHP model to evaluate all eligible nodes (closer to the destination location) in its communication range based on the parameters of each node: distance to the destination, remaining battery capacity, and queue size. The source node chooses the top $M$ nodes based on the local weight that this node will be selected. The source node sends a Route Acknowledgement (RA) packet to each desired node, and each desired node will reply using a REPLY packet if it is available. The structure of RA and REPLY is summarized in Table 3. If after a certain period of time, the source node did not receive REPLY from some desired node, it will pick the node with highest weight among the remaining $N-M$ ndoes. In the second hop, the selected node in each path will choose its next hop node using the same process. As illustrated in Fig. 2, node B needs to choose one node from four eligible nodes C, D, E, and F based on their three parameters, and sends RA packet to the selected node and waits for REPLY. If the top one node is unavailable (for exmaple, selected by another path), then the top second node will be selected. Consequently, $M$ paths can be set up. 
Table 3. RA and REPLY message structure

\begin{tabular}{l|l|l|l|l|l}
\hline Type & Desired Node ID & Self Node ID & Dest_X & Dest_Y & Src_ID \\
\hline
\end{tabular}

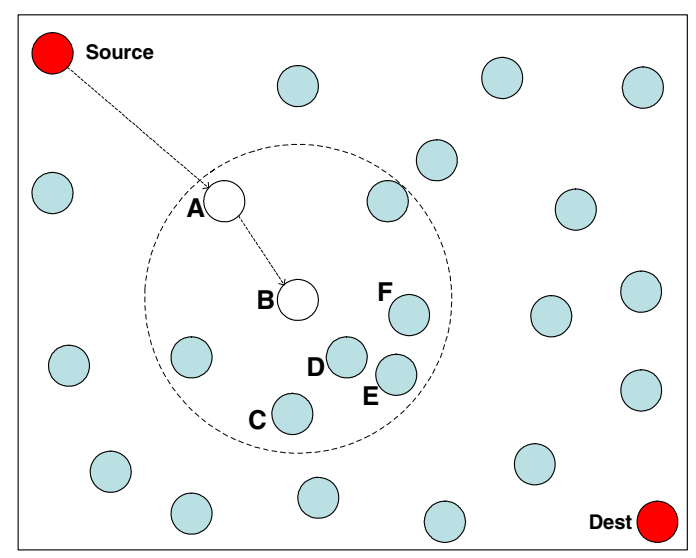

Fig. 2. Illustration of next hop node selection

\section{Performance Evaluations}

\subsection{Simulation Environment}

In order to evaluate the nodes selection in multipath routing by AHP, we have used JSim [15] as the simulation environment. 60 sensors are randomly deployed in an area of $100 \mathrm{~m} \times 100 \mathrm{~m}$. The source and destination sensors are set as $2 \mathrm{~J}$ initially, and $5 \mathrm{cou}-$ ples of source and destination nodes are communicating at the same time in this network. All the other sensors have initial energy of 0-2J. The buffer capacity of each sensor node has been taken as 5 packets with packet length 512 bit and bit rate $9.6 \mathrm{~kb} / \mathrm{sec}$. The time interval $T$ is set as $10 \mathrm{~s}$ in our simulation. The source node select $M=3$ nodes in its communication range for the first hop relay. From the second hop, each node along the 3 paths selects only one node toward its next hop.

In AHP modeling, the matrix A is determined as follows according to Section 3:

$$
\begin{aligned}
& \begin{array}{ccc}
\begin{array}{c}
\text { Distance } \\
\text { to Dest }
\end{array} & \begin{array}{c}
\text { Residual } \\
\text { energy }
\end{array} & \begin{array}{c}
\text { Queue } \\
\text { size }
\end{array}
\end{array} \\
& \text { ( } \alpha) \quad(\beta) \quad(\gamma) \\
& A=\begin{array}{l}
\alpha \\
\beta \\
\gamma
\end{array}\left[\begin{array}{ccc}
1 & 2 / 1 & 3 / 1 \\
1 / 2 & 1 & 2 / 1 \\
1 / 3 & 1 / 2 & 1
\end{array}\right]
\end{aligned}
$$

where the three criteria are denoted by $\alpha, \beta$ and $\gamma$ respectively. 


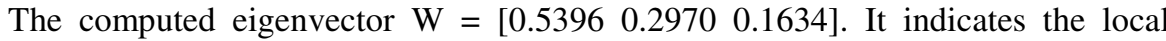
weight of the distance to destination, remaining battery capacity, and queue size, respectively, so that we can see clearly that the distance to destination is the most important criterion, and queue size is the least. According to Eq. (6), we can get the eigenvalue $\lambda_{\max }=3.0093$. Consequently, consistency ratio can be calculated as $C R=$ $0.0047<0.1$, thus matrix A satisfies the consistency check.

Each sensor node determines the weight matrixes of alternatives under three factors and then gets global weight based on its specific situation. Its eligibility as next hop relay node can be finally decided by the AHP hierarchy model.

\subsection{Simulation Results}

We compare our AE-GMR against the geographical multipath routing (GMR) [11] scheme where only distance to the destination is considered. In Fig. 3, we plot the simulation time versus the number of nodes dead. It shows that when $50 \%$ nodes (30 nodes) die out, the network lifetime for AE-GMR has been extended about $40 \%$. In

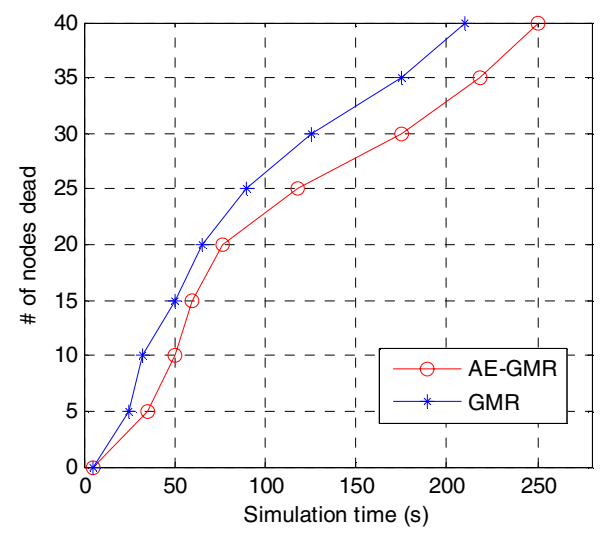

Fig. 3. Lifetime comparison

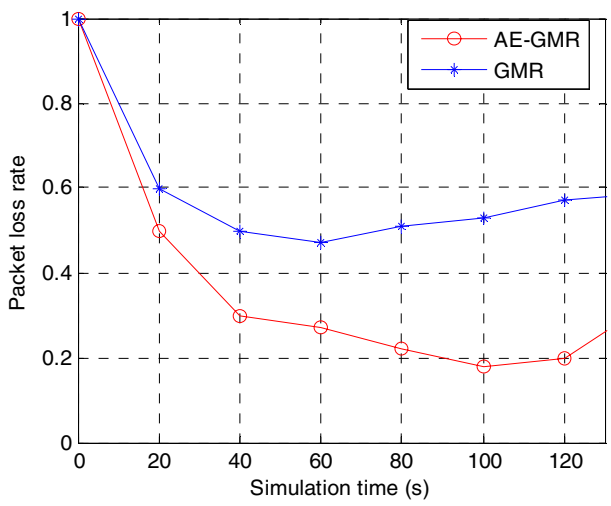

Fig. 4. Simulation time vs. packet loss rate 
Fig. 4, we compare the packet loss rate of these two schemes. Packets are dropped either due to insufficient buffer capacity at the receiver or because of the lack of energy needed to transmit the packet. Observe that our AE-GMR outperforms the GMR with about $20 \%$ less packet loss resulting in greater reliability. The average latency during transmission (end-to-end) is $422.16 \mathrm{~ms}$ for our AE-GMR and $407.5 \mathrm{~ms}$ for GMR, and link failure rate for AE-GMR is $6.24 \%$, but for GMR is $10.42 \%$.

\section{Conclusion and Future Work}

In this paper, we proposed an energy aware geographical multipath routing scheme for WSNs. Three factors contributing to the next hop relay node selection are considered and they are the distance to the destination location, remaining battery capacity, and queue size of candidate sensors in the local communication range, respectively. Analytical Hierarchy Process (AHP) was applied for optimal decision making. We evaluated the efficiency of our proposed scheme and the simulation results showed that this scheme could extend the network lifetime longer than the original geographical routing scheme which only considered distance to the destination location. Moreover, the proposed scheme could reduce the packet loss rate and link failure rate since the buffer capacity was considered.

In the future work, we may consider the node mobility as another factor for routing decision making and design such routing protocol.

Acknowledgments. This research was supported by the MIC (Ministry of Information and Communication), Korea, under the ITRC (Information Technology Research Center) support program supervised by the IITA (Institute of Information Technology Advancement) (IITA-2006-C1090-0602-0002).

\section{References}

1. Wu, X., Heo, H., Shaikh, R.A., Cho, J., Chae, O., Lee, S.: Individual Contour Extraction for Robust Wide Area Target Tracking in Visual Sensor Networks. In: Proc. of 9th IEEE International Symposium on Object and component-oriented Real-time distributed Computing (ISORC), Gyeongju, Korea, pp. 179-185 (2006)

2. Bhagwat, C.P.: Highly dynamic destination-sequenced distance vector routing. In: Proc. of ACM SIGCOMM, pp. 234-244. ACM Press, New York (1994)

3. Chiang, C.-C., Wu, H.-K., Liu, W., Gerla, M.: Routing in clustered multihop mobile wireless networks with fading channel. In: Proc. IEEE Singapore Intl Conference on Networks (1997)

4. Perkins, C.E., Royer, E.: Ad hoc on demand distance vector routing. In: Proc. 2nd IEEE Workshop o Mobile Computing Systems and Applications, IEEE Computer Society Press, Los Alamitos (1999)

5. Johnson, D., Maltz, D.: Mobile Computing. Kluwer Academic Publishers, Dordrecht (1996)

6. Lee, S.J., Gerla, M.: Split Multipath Routing with Maximally Disjoint Paths in Ad Hoc Networks. ICC (2001) 
7. Wang, L., Shu, Y.T., Dong, M., Zhang, L.F., Yang, W.W.: Multipath Source Routing in wireless Ad Hoc Networks. In: Canadian Conference on Electrical and Computer Engineering, vol.1, pp. 479-483 (2000)

8. Nasipuri, A., Das, S.R.: On-Demand Multipath Routing for Mobile Ad Hoc Networks. In: IEEE ICCCN, pp. 64-70. IEEE Computer Society Press, Los Alamitos (1999)

9. Finn, G.G.: Routing and addressing problems in large metropolitanscale internetworks. USC ISI Report ISI/RR-87-180 (1987)

10. Xing, G., Lu, C., Pless, R., Huang, Q.: On Greedy Geographic Routing Algorithms in Sensing-Covered Networks. In: Xing, G., Lu, C., Pless, R., Huang, Q. (eds.) ACM International Symposium on Mobile Ad Hoc Networking and Computing (MobiHoc), Japan (2004)

11. Jain, R., Puri, A., Sengupta, R.: Geographical routing using partial information for wireless sensor networks. In: IEEE Personal Communications, pp. 48-57 (2001)

12. Heinzelman, W.B., Chandrakasan, A.P., Balakrishnan, H.: An Application-Specific Protocol Architecture for Wireless Microsensor Networks. IEEE Transactions on Wireless Communications 1(4), 660-670 (2002)

13. Saaty, T.L.: The Analytic Hierarchy Process. NY, McGraw Hill (1980)

14. Song, Q.Y., Jamalipour, A.: A network selection mechanism for next generation networks. In: IEEE Int. Conf. Communication (ICC), vol.2, pp. 1418-1422 (2005)

15. J-Sim, http://www.j-sim.org/ 